\title{
Características de las luciérnagas: determinación de la intensidad de emisión luminosa
}

\author{
Ignacio Escobar Mejía ${ }^{1, \psi}$ \\ ${ }^{1}$ Ingeniero Electricista. Medellín, Colombia
}

Recibido 4 de abril de 2019. Aceptado 29 de julio de 2019

\begin{abstract}
Resumen-Se estudian las diferentes características que presentan las luciérnagas, comenzando por su clasificación taxonómica, la producción de la luz por los insectos y las unidades de iluminación del Sistema Internacional de Unidades. Se analizan los diferentes valores de luminosidad encontrados por otros autores y se describe un método sencillo para determinarla utilizando equipos accesibles en cualquier laboratorio básico electrónico. De un total de 154 insectos capturados, 53 probablemente fueron hembras y producen valores de luminosidad iguales a 3,6 milicandelas (mcd); 82 insectos probablemente machos producen una luminosidad igual a 2,2 mcd. Se encuentran otros 19 ejemplares inciertos cuyo patrón de iluminación no pudo definirse claramente.
\end{abstract}

Palabras clave - Luciérnagas: Luciferasa: Luciferina: Milicandela.

\section{Characteristic Of The Fireflies. Determination Of The Intensity Of Light EMISSION}

\footnotetext{
Abstract - Different characteristics presented by fireflies are studied, such as their taxonomic classification, light production and the luminous units of the International System of Units. Different intensity values found by other authors are analyzed and a simple method to determine it, using accessible equipment available in any basic electronic laboratory, is described. Of a total 154 captured insects, probably 53 were females and produced brightness values equal to 3.6 millicandelas (mcd); 82 insects, probably male, produced values equal to $2.2 \mathrm{mcd}$. There are 19 other specimens whose uncertain illumination pattern could not be clearly defined.
}

Keywords - Firefly; Luciferase; Luciferin; Milicandela. 


\section{INTRODUCCIÓN}

\subsection{Generalidades}

Tas luciérnagas son pequeños insectos voladores de forma ligeramente ovalada, color café y presentan rayas amarillas, las cuales cruzan a lo largo del animal; miden aproximadamente entre 5-20 milímetros $(\mathrm{mm})$ de largo y tienen la capacidad de emitir luz fría con una eficiencia cercana al $100 \%$ [1]. Algunos ejemplares pequeños no tienen el órgano luminiscente [2]. Están presentes en casi todas las regiones tropicales y sub-tropicales del mundo, menos en Australia y Nueva Zelanda y se calcula en más de 2.000 las especies existentes dentro de los 359.891 coleópteros registrados para un total de 1.004 .898 insectos $[3,4]$.

\subsection{Clasificación taxonómica}

Su clasificación taxonómica puede resumirse así:

Reino: animal. Filo: artrópodos. Clase: insectos. Orden: coleópteros. Superfamilia: Elateroidea. Familia: Lampyridae. Género: ------. Especie: ------.

Las clasificaciones de Género y Especie que se encuentran en el territorio colombiano han sido poco estudiadas. Zaragoza [5] clasifica ocho especies de la Bicellonycha Motschulsky en Bolivia, Brasil, Colombia, Paraguay, Perú y Venezuela; Zaragoza Caballero Santiago, [6] clasifica como especie la Bicellonycha colombiana perteneciente al Género Bicellonycha (1989); el mismo autor [7], clasifica un ejemplar capturado en la localidad de Calima, Valle del Cauca, Colombia, bajo el Género Bicellonycha y con nombre específico de Bicellonycha stigmatica. Motschulsky [8], clasifica los insectos bajo el Género Photinus y como Especie Photinus cinctellus y están presentes en México, Guatemala, Nicaragua, Costa Rica Argentina y Colombia. Zaragoza Caballero y Pérez Hernández [9], describen otra familia de insectos luminiscentes llamada Pengodidae compuesta por 34 Géneros y 263 especies ubicados exclusivamente en el Continente Americano. Clasifican 26 especies en Colombia, pero ninguna de ellas hace parte del trabajo.

\subsection{Producción de luz}

El proceso por el cual las luciérnagas producen la luz es 1lamado Bioluminiscencia. En los adultos, el órgano productor de la luminosidad se denomina Photurus [10] y está situado en el sexto y séptimo segmentos ventrales; se compone por 3 capas diferentes a saber:

1. Una capa exterior transparente.

2. El órgano luminiscente, portador de las células productoras de luz (Fotocitos) que están llenas de grandes y pequeños gránulos negros. Los gránulos grandes contie- nen moléculas emisoras de luz llamadas Luciferina y los pequeños son mitocondrias, fuente de ATP necesario para la reacción productora de luz [11].

3. Una capa opaca que le sirve de reflector y está formada, principalmente, por cristales de ácido úrico [11].

Para que pueda darse el fenómeno luminiscente se necesitan 4 variables:

- Un compuesto químico, Luciferina.

- Una enzima, la luciferasa, también presente en la misma pared del órgano luminiscente y actúa como catalizadora de la reacción.

- Moléculas de ATP (Trifosfato de Adenosina), productoras de la energía.

- Oxígeno, el cual es transportado hasta el órgano bioluminiscente por un sistema similar a los pulmones y su cantidad aportada influye en la intensidad de luz producida $[11,12,13]$.

La reacción de la luciferina con el oxígeno produce una sustancia llamada oxiluciferina, la cual contiene electrones en estado excitado mientras ocurre la reacción; al corto tiempo, estos electrones van a su estado estable liberando fotones de luz.

\subsection{Unidades de iluminación}

\subsubsection{La Candela (I, Intensidad luminosa, cd)}

La Candela se define como: "La intensidad luminosa en una dirección dada, de una fuente que emite una radiación monocromática de frecuencia $540 \times 10^{12} \mathrm{~Hz}$ y de la cual la intensidad radiada en esa dirección es $1 / 683 \mathrm{~W}$ por estereorradián" [14]; de forma aproximada, corresponde a la iluminación producida por una vela de esperma de 20 $\mathrm{mm}$ de diámetro y una altura de llama igual a $50 \mathrm{~mm}$ [15].

\subsubsection{El Lumen (F, Flujo luminoso, $\mathrm{lm}$ )}

Se define como: "Unidad de flujo luminoso del Sistema Internacional de Unidades, que equivale al flujo luminoso emitido por una fuente puntual uniforme situada en el vértice de un ángulo sólido de un estereorradián y cuya intensidad es una candela" [16].

1.5. Valores de iluminación reportados por varios autores

Coblentz, (1912) [17] encuentra en la especie de luciérnagas, la Photinus Pyralis, que produce 1/50 de Candela (20 milicandelas) (mcd) y la registra como la máxima intensidad encontrada. Harvey (1952) [18], consigue en la Pyractomena Borealis (Familia Lampyridae) una intensidad lumínica de 9/50 de Candela (180 mcd). 


\subsection{Otras características de las luciérnagas}

El proceso mediante el cual se produce la iluminación es controlado por su sistema nervioso, y la frecuencia de aparición de las ondas varía según sea un macho o una hembra. El color de la emisión es amarillo - verdoso y su longitud de onda ha sido apreciada por Seliger [19], en un intervalo que va de los $552 \mathrm{~nm}$ a los $582 \mathrm{~nm}$.

Las luciérnagas producen la iluminación aparentemente para estos propósitos: el primero, para localizar el sitio de "aterrizaje" de las hembras con el fin de encontrar un sitio adecuado para poner sus huevos. Segundo, como medio de comunicación entre machos y hembras con fines copulativos [20]; los diferentes patrones de iluminación, permiten un ambiente en donde conviven varias especies el encontrarse los miembros de la misma y definir el sexo del contrario o bien, la hembra cambia su patrón al ver a otro macho diferente a su especie para atraerlo, y una vez cae sobre ella, lo devora; éste es el fenómeno conocido como: "La femme fatale" [21, 22, 23].

Las luciérnagas parecen ser carnívoras [24]. También se describe una especie, la Photinus Macdermotti en la que el macho se acerca a la luz producida por la hembra aterrizando cerca de ella y luego camina hacia ésta para cerciorarse que es de su misma especie y así prevenir el ser devorado [25]. Normalmente, las hembras se encuentran rastreras por entre la vegetación [26], mientras los machos vuelan a una altura no mayor de 6 metros buscando la iluminación producida por las hembras. Algunos autores también describen el fenómeno de la sincronización entre los insectos voladores $[3,27]$.

El trabajo pretende mostrar un método sencillo para medir la intensidad luminosa de las luciérnagas, tomando como estándar la medida propuesta por el Sistema Internacional de Unidades, la candela.

\section{Materiales y Métodos}

\subsection{Equipo necesario}

- Una esfera integradora de luz, de $247 \mathrm{~mm}$ de diámetro, construida por el autor.

- Un generador de funciones capaz de suministrar una onda cuadrada de 50 milisegundos (ms) y de una amplitud y frecuencia variables, construido por el autor.

- Un diodo LED de referencia, marca Panasonic, modelo LN48YCP, color ámbar cuyo diámetro es de $3 \mathrm{~mm}$.

- $\quad$ Resistencias eléctricas de $10 \Omega$ y de $100 \Omega, 5 \%$ $\mathrm{y}^{1 / 4} / 4$ de vatio $(\mathrm{W})$.
- Un fotodiodo de silicio marca Hamamatsu, modelo S1337-1010BR.

- Un amplificador de transimpedancia, construido por el autor.

- Un osciloscopio digital marca BK Precision, modelo 2540 .

- Solución al $20 \%$ de detergente Extran ${ }^{\circledR}$ (Merck MA 01.)

- Una memoria USB

- Un cazamariposas.

\subsection{Configuración y operación del sistema}

En la Fig. 1 se presenta un esquema de la configuración del instrumento usado para registrar la iluminación producida por las luciérnagas.

\subsubsection{Esfera integradora de luz}

La esfera integradora de luz (Fig. 2) consta de dos semiesferas y está construida con acrílico termoplástico (polimetilmetacrilato) (Acrílicos JL, Medellín, Colombia) con un espesor de $3 \mathrm{~mm}$, y tiene un diámetro interno igual a $247 \mathrm{~mm}$. El diámetro se escoge según criterios dados por varios autores $[28,29]$. El interior está pintado con esmalte aerosol blanco mate (Aerosol multiusos, ref. 7205030, Compañía Pintuco S.A., Medellín, Colombia). Algunos [30] recomiendan recubrirlo con Sulfato de Bario (Sulfato de Bario de 0,7 $\mu \mathrm{m}$, Sachtleben Chemie Gmbh., Duisburg Germany) pero en el estudio no funcionó por contaminarse la luciérnaga con dicho polvo y no producir la iluminación.

Las pruebas de reflectancia de la esfera fueron hechas con un colorímetro "ColorEye" Gretag - Macbeth 700 DA y arrojaron los siguientes valores:

- Esfera recubierta con sulfato de bario: $90,6 \%$

- Esfera pintada con esmalte aerosol blanco mate: $88,6 \%$

Lo anterior indica que todos los valores de luminosidad reales son un poco mayores que el valor experimental obtenido.

La esfera lleva 3 orificios: uno, lleva el diodo LED de calibración; otro, el fotodiodo captador de la iluminación; y un tercero, un visor para comprobación. La superficie total de los orificios es un 3,21\% del total del área interior de la esfera. Se recomienda tener dicho valor inferior al 5 $\%$ del área total de la esfera [31].

El exterior de la esfera está pintado con 3 capas de esmalte aerosol negro mate (Aerosol multiusos, ref. 7580030, Compañía Pintuco S.A., Medellín, Colombia) para prevenir la entrada de luz extraña a la esfera. 


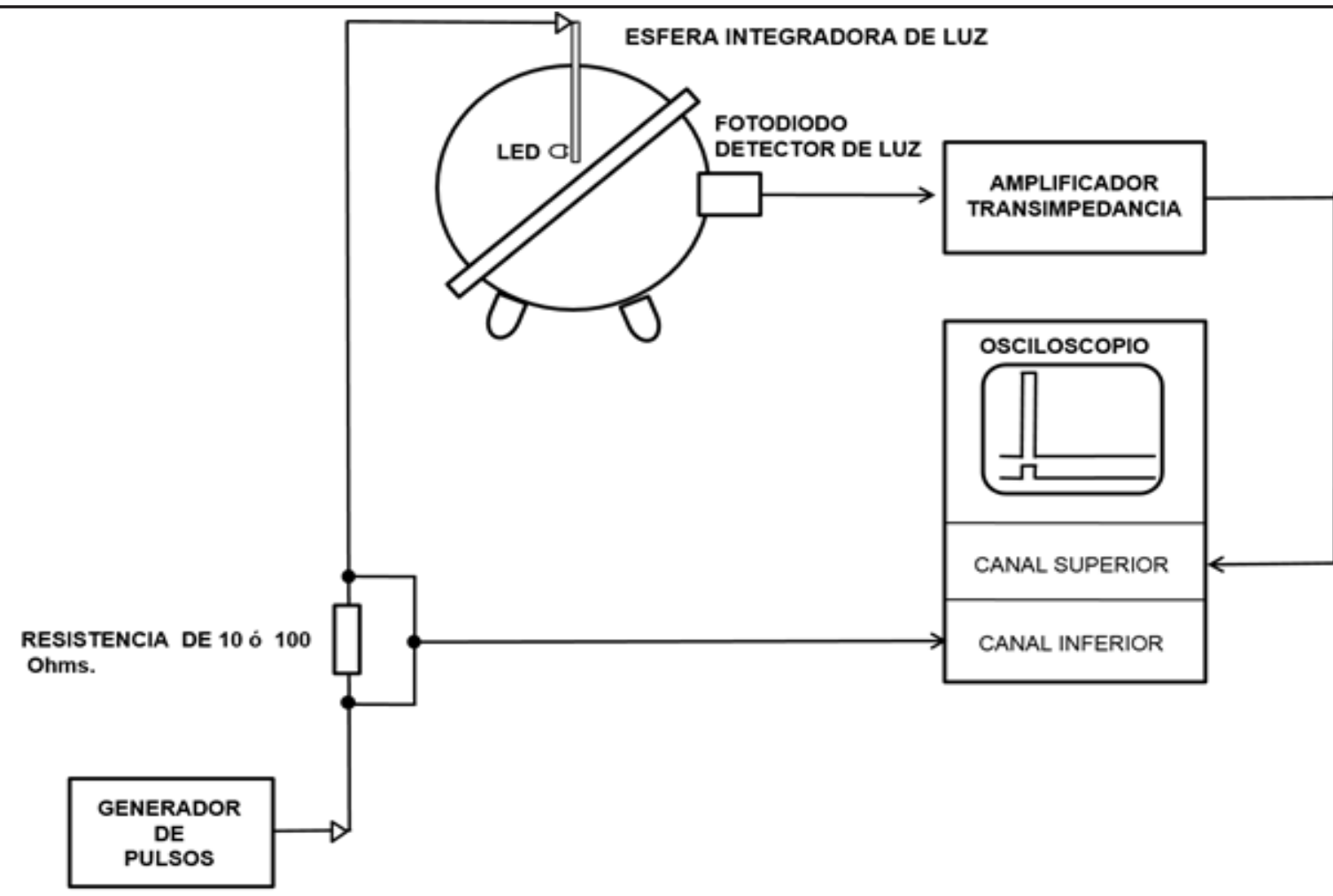

Fig. 1. Configuración del instrumental para registrar la iluminación producida por las luciérnagas

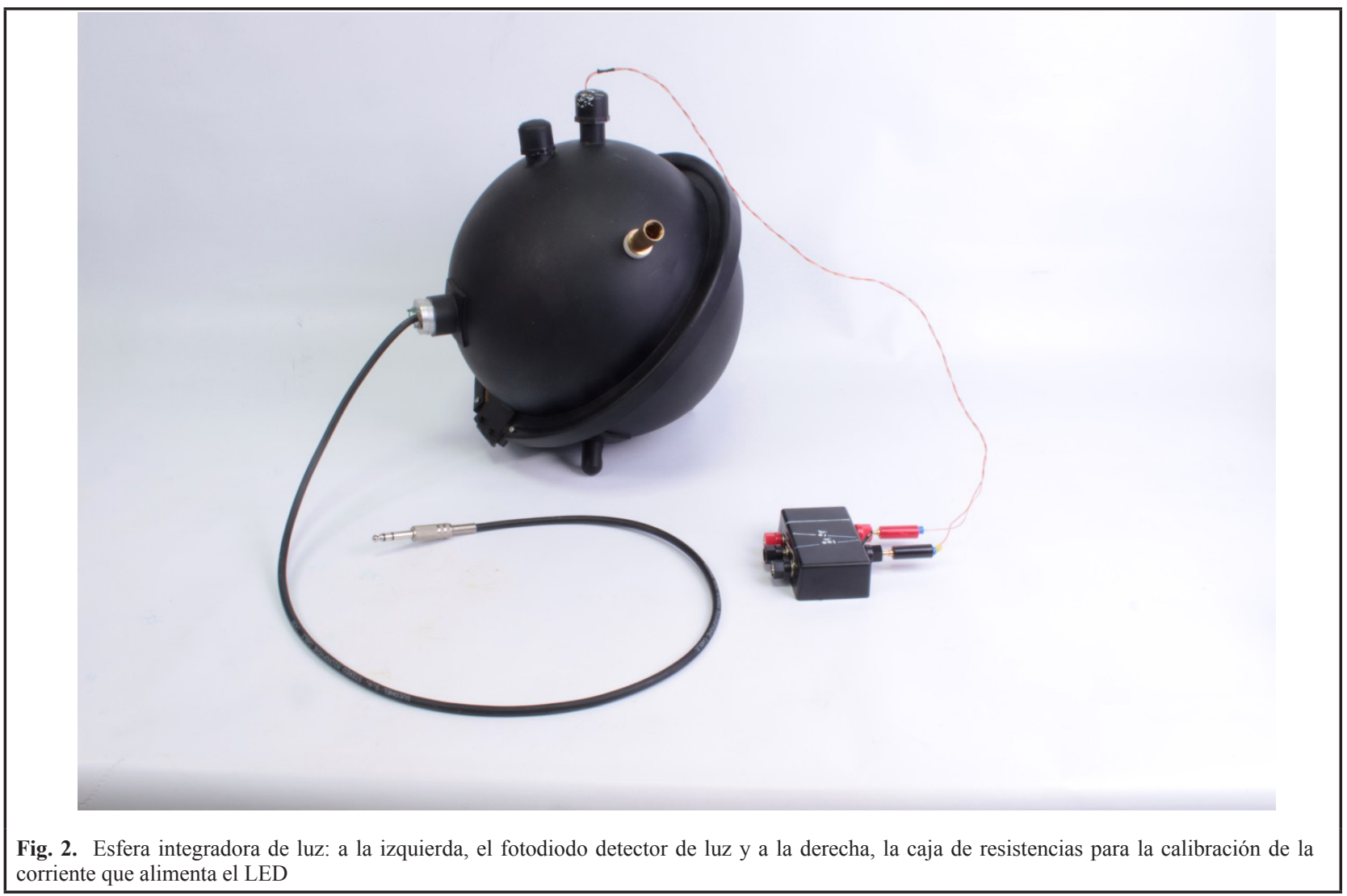




\subsubsection{Generador de pulsos}

Está constituido por 2 etapas de circuitos integrados, tipo NE 555. La primera etapa produce la frecuencia de operación, y la segunda etapa provee la duración del pulso.

Se elige un pulso de 50 (ms) de duración y su repetición se controla a voluntad. La duración se escogió por error y tanteo luego de observar en el osciloscopio la duración de los pulsos luminosos producidos por las luciérnagas.

\subsubsection{Diodo LED de referencia}

Un diodo LED de $3 \mathrm{~mm}$ de diámetro color ámbar cuya longitud de onda es de $585 \mathrm{~nm}$. Su propósito es servir como calibrador para conocer la iluminación producida por la luciérnaga. Se alimenta por medio del generador de pulsos a través de la resistencia para la calibración. El LED está colocado en el centro de la esfera en tal forma que no ilumine directamente al fotodiodo detector de la iluminación. De acuerdo con la hoja de características del LED LN48YCP, se presenta una relación lineal entre la corriente y la intensidad, donde una corriente entre 2 y 40 $\mathrm{mA}$ produce una intensidad luminosa entre 0,3 y $10 \mathrm{mcd}$, respectivamente [32].

\subsubsection{Resistencia de $10 \Omega$}

En los primeros experimentos con 109 insectos se aplicaron 20 miliamperios (mA) para estimular el LED y obtener así las 6 mcd como referencia de la iluminación. Se procedió a conectar una resistencia de $10 \Omega$ en serie con la línea de alimentación del LED y obtener una caída de tensión igual a 200 milivoltios $(\mathrm{mV})$ en bornes de dicha resistencia. Esa caída de tensión se aprecia en el canal $\mathrm{N}^{\circ} 2$ del osciloscopio y se toma como referencia para la calibración de la iluminación.

\subsubsection{Resistencia de $100 \Omega$}

En cambio, para los últimos 45 experimentos se estimuló el LED con una corriente mucho menor $(5 \mathrm{~mA})$ para obtener así $1 \mathrm{mcd}$ en la iluminación del LED. Con esta disposición se pretende conseguir una mayor estabilidad en el valor de iluminación del LED de referencia. Al pasar una corriente de $5 \mathrm{~mA}$ a través de ella, proporciona una caída igual a $500 \mathrm{mV}$ la cual es observada en el canal 2 del osciloscopio. Otra característica importante en utilizar la última disposición es el poder tener la caída de tensión mayor $(500 \mathrm{mV})$ y proporcionar una correcta sincronización (Trigger) del osciloscopio al aparecer la señal producida por la luciérnaga.

\subsubsection{Fotodiodo de silicio}

El fotodiodo de silicio marca Hamamatsu modelo S1337-1010BR [33] presenta una superficie activa de 100 $\mathrm{mm}^{2}$ y una banda espectral entre los $320 \mathrm{~nm}$ y los 1.100 $\mathrm{nm}$. Va montado en un soporte cilíndrico de aluminio y sirve para detectar la iluminación; la superficie del sensor está cubierta por una placa cubreobjetos para microscopio ( $\mathrm{N}^{\circ}$ 1. de $0,14 \mathrm{~mm}$ de espesor) con el fin de protegerlo de las rayas producidas por las uñas que las luciérnagas tienen en sus patas.

\subsubsection{Amplificador de señal}

El propósito del amplificador es el de servir como acoplamiento entre el foto-diodo y el osciloscopio, así como también para obtener la amplitud requerida de la onda observada y trabaja como amplificador fotovoltaico. Está conformado por un sistema compuesto por 2 etapas a saber:

- Un amplificador operacional de alta impedancia de entrada $\left(10^{12} \Omega\right)$ JFET TL071 [33] que presenta bajo nivel de ruido.

- Un segundo amplificador operacional convencional para el ajuste de la ganancia fina dada por medio de un potenciómetro de precisión (Helipot $\left.{ }^{\circledR}\right)$ de 10 Kohms colocado en su circuito de realimentación.

\subsubsection{Osciloscopio}

Se utilizó un osciloscopio digital de 2 canales con una respuesta de 60 Megahertz $(\mathrm{MHz})$ y una rata de muestreo igual a 1 Giga-muestras/segundo $(\mathrm{GSa} / \mathrm{s})$. En el canal $\mathrm{N}^{\circ} 1$ se observa la iluminación producida, bien sea por la señal de calibración, o por la iluminación producida por el insecto. En el canal $\mathrm{N}^{\circ} 2$ se mide la corriente de alimentación del LED necesaria para la calibración.

\subsection{Medición de la luminosidad en las luciérnagas}

\subsubsection{Campo de observación}

El campo de observación está situado en el Municipio de Caldas, Departamento de Antioquia, República de Colombia (Coordenadas: N 06 03 ' 3265': W $075^{\circ} 38^{\prime}$ 07.4"). La temperatura exterior (promedio de 64 mediciones) fue de $19,4{ }^{\circ} \mathrm{C}$, y la interior en el laboratorio (promedio de 95 mediciones) fue de $21,1^{\circ} \mathrm{C}$. La humedad relativa exterior (promedio de 63 mediciones) fue de $61 \%$. Todas las medidas fueron tomadas a las 18:30. En época lluviosa no se observa actividad alguna y la iluminación externa también contribuye a alejarlos; lo mismo sucede cuando la luna está llena. 


\subsubsection{Cuidado de los animales}

El cuidado y disposición de los insectos se llevó a cabo según las indicaciones de las normas nacionales e internacionales $[35,36]$.

\subsubsection{Limpieza de la esfera}

Antes de introducir el insecto dentro de la esfera, esta se limpia con una solución al $20 \%$ de detergente Extran ${ }^{\circledR}$ MA 01 (Merck KGaA, Darmstadt, Alemania). Ello se hace con el fin de quitarle los residuos dejados por el anterior insecto introducido. Además, cuando la esfera se encuentra sin uso, se introduce en ella un poco de hierba húmeda para que así produzca un olor natural al ambiente en que se encuentran los insectos.

\subsubsection{Calibración del sistema}

Luego de dejar estabilizar el equipo durante $1 / 2$ hora, se procede a revisar el estado de carga de las baterías y también calibrar el sistema. En los dos canales de la pantalla del osciloscopio se pueden observar:

- $\quad$ En el canal $\mathrm{N}^{0} 1$, de color amarillo, se observa la respuesta del amplificador al estímulo producido por la iluminación del LED de calibración o a la respuesta producida por la luciérnaga.

- En el canal $\mathrm{N}^{\mathrm{o}} 2$, color azul, se aprecia el pulso de calibración, o sea la caída de voltaje a través de la resistencia de $10 \Omega$ o bien en la de $100 \Omega$ que corresponden a 6 mcd o bien a $1 \mathrm{mcd}$.

\subsubsection{Captura de los insectos}

Los insectos se capturan entre las 18:30 y las 21:00. Durante este tiempo se presentan el mayor número de luciérnagas. La caza de las luciérnagas se hace por medio de un cazamariposas o "Gama Británica" de $40 \mathrm{~cm}$ de diámetro [37]. Se observa cuando iluminan y se capturan de a una cada vez, teniendo extremo cuidado de no molestarlas, pues de lo contrario no producirán luminosidad luego de introducirlas en la esfera. Se capturan aquellos que están volando entre los $0,50 \mathrm{~m}$ y los $3,0 \mathrm{~m}$ a partir del nivel del suelo y se supone que estos son machos [38]. Los insectos así capturados se denominan como "Voladores". Las luciérnagas hembras van por el follaje de la hierba, y se denominan "Rastreros".

\subsubsection{Introducción del insecto en la esfera integradora}

La luciérnaga capturada se introduce en la esfera integradora de luz y allí se deja durante media hora en total oscuridad, sin proceder a estimular con la iluminación del diodo LED de referencia. Si se produce una respuesta, se graba en la memoria USB conectada al osciloscopio. Luego de transcurrir media hora sin haber obtenido iluminación alguna se procede a estimular con un pulso de corriente de $50 \mathrm{~ms}$ de duración para observar si existe la sincronización.

Si la luciérnaga ilumina, se observa su respuesta en el canal $\mathrm{N}^{\circ} 1$ del osciloscopio. Es muy importante mantener la altura del pulso de calibración, pues con ello se garantiza la veracidad de la medición. Se hacen las anotaciones correspondientes en la bitácora tomando también la hora de aparición de la respuesta.

\section{Resultados y DiscusióN}

\subsection{Insectos capturados}

Se capturaron 154 ejemplares y de ellos se tomaron 1.016 registros de su actividad luminosa. De ellos, 82 fueron voladores y 53 rastreros. Se capturaron 19 cuyo sexo no se pudo suponer. Se anotó el sexo supuesto del insecto, la hora de la producción de la actividad luminosa, el número de la imagen vista en el osciloscopio y algún dato que pudiera interesar.

\subsubsection{Características del pulso}

Saikia et al [39], describen el pulso de luz en forma de espiga similar a una onda triangular de base muy pequeña. Sus registros fueron tomados con un registrador a papel a una velocidad de $25 \mathrm{~cm}$ por minuto. Lloyd y Ballantyne [40], utilizan un sistema de fotomultiplicador y un registrador de cinta magnético. Sus registros son más similares a los del presente trabajo.

La forma de la señal luminosa está conformada por ondas simétricas con respecto a su eje central, bastante parecidas a la "campana de Gauss" excepto con una caída un poco lenta al final de la onda, característica típica de los procesos biológicos.

\subsubsection{Respuesta de las hembras}

Se ha observado que los 69 insectos hembras (53 insectos capturados como "Rastreros" y 16 capturados como "Voladores" pero que alumbraron como hembras) producen 2 o máximo 3 respuestas. La amplitud de la onda (promedio de 65 registros) fue de 3,6 mcd y la duración (promedio de 65 registros) medida en la base de la onda fue de 98,8 ms (Fig. 3). 


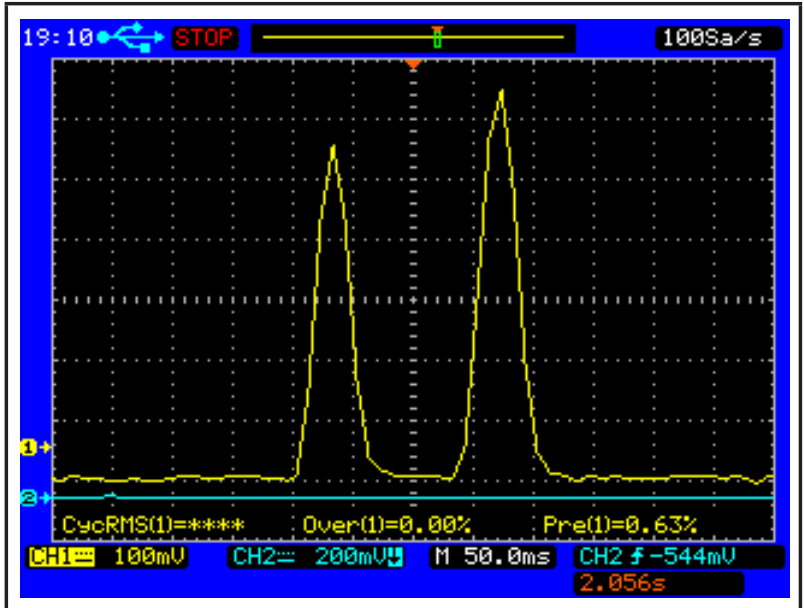

Fig. 3. Ondas registradas en una luciérnaga supuestamente hembra capturada en Caldas, Antioquia, Colombia. Fecha, 101018. Ejemplar $\mathrm{N}^{\circ} 25$ - 1. Registro $\mathrm{N}^{\mathrm{a}}$ LC 184. Calibración vertical, 1 $\mathrm{mcd}=100 \mathrm{mV}$. Puede notarse a caída un poco lenta al final de la pendiente de bajada

\subsubsection{Respuesta de los machos}

Los ejemplares supuestamente machos producen trenes de pulsos compuestos por ondas similares a las de las hembras, pero que se repiten en el número de ondas hasta de ocho veces. Casi siempre empiezan con amplitud creciente y terminan con ondas de menor amplitud (Fig. 4).

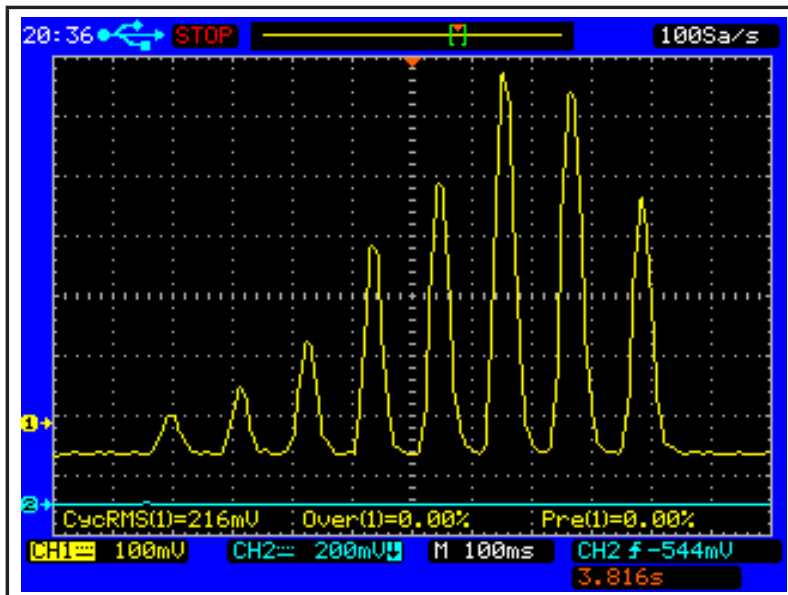

Fig. 4. Tren de pulsos producidos por un ejemplar supuestamente macho. Registro tomado en Caldas, Antioquia, Colombia. Fecha, 10 10 18, Ejemplar No 25 - 2, registro LC 223. Calibración vertical, 1 $\mathrm{mcd}=100 \mathrm{mV}$

Se capturaron 82 ejemplares machos "Voladores" y 2 "Rastreros" (pero que presentaban el patrón de iluminación como machos). La amplitud promedia de las ondas (promedio de 96 registros) fue de 2.2 mcd y la duración medida en la base de las ondas (promedio de 59 registros) fue de 86 ms. Se tomaron valores por encima de $0,1 \mathrm{mcd}$ para calcu- lar los resultados. Nótese como el valor promedio para los machos es mucho menor que el de las hembras.

\section{CONCLUSIÓN}

En el presente trabajo se pretende de manera muy sencilla, reproducir con bastante fidelidad la respuesta luminosa de las luciérnagas de la familia Lampyridae. Se encuentran valores ajustados que van desde un mínimo detectado de 0,02 med (Ejemplar LCG34) hasta las 10,6 mcd (Ejemplar L898d). Debe notarse que todos los valores de luminosidad encontrados en el presente trabajo fueron ajustados a la reflectancia de la esfera, que es de $88,6 \%$, Es decir, los valores obtenidos se multiplicaron por un factor de 1.114.

\section{Agradecimiento}

El autor agradece al Laboratorio de Color de la Compañía Pintuco S. A. por haber facilitado su colorímetro "ColorEye" Gretag - Macbeth 700 DA. Al Ingeniero Pablo José Mejía O., Gerente de I + D + i de Dies S. A. por haber suministrado el fotodiodo Hamamatsu.

\section{REFERENCIAS}

[1]. White, R.E. Peterson field guides: "Beetles". Houghton Mifflin Co., 1998, pp. 188-191.

[2]. Borror D.J., De Long D.M. An introduction to the study of insects. Barnes \& Noble, 1992. Capítulo 28, 432.

[3]. Buck J., \& Buck E. (1976). Synchronous fireflies. Scientific American, 234 (5), 74-85.

[4]. Bouchard P., Grebennikov V.V., Smith ABT, Douglas H (2009) Biodiversity of Coleoptera [pp. 265-301]. En: Foottit RG, Adler $\mathrm{PH}$ (Eds). Insect biodiversity: science and society. Blackwell Publishing, Oxford, 656, Consultado el 30 de Abril de 2003 en: http://www.lacbiosafety.org/wp-content/uploads/2011/09/insectbiodiversity-science-and-society1.pdf

[5]. Zaragoza-Caballero S. Trece especies nuevas de Bicellonycha Motschulsky, 1852 (Coleoptera: Lampyridae, Photurinae) de América. Anales del Instituto de Biología. Universidad Nacional Autónoma de México, 59 (2), 253-286. 1989.

[6]. Zaragoza. Bicellonycha colombiana, 1989. Consultado el 5 de diciembre de 2015 en: http://insecta.pro/taxonomy/971438

[7]. Zaragoza-Cabellero S. Bicellonycha stigmatica - IBUNAM: CNIN: COL-9266". UNIBIO: Colecciones Biológicas. 2008-0403. Universidad Nacional Autónoma de México. Consultado el 5 de diciembre de 2015 en: http://unibio.unam.mx/ collections/ specimens/urn/IBUNAM:CNIN:COL-9266

[8]. Motschulsky, 1854 Photinus cinctellus, Taxonomic serial $\mathrm{N}^{\circ}$ 952851, Integrated taxonomic information system -ITIS- 
Consultado el 5 de diciembre de 2015 en: http:// http://www.itis. gov/servlet/SingleRpt/Sin

[9]. Zaragoza-Caballero S., Pérez C.X. Sinopsis de la familia Phengodidae (Coleoptera): trenecitos, bigotudos, glow-worms, railroad-worms o besouros trem de ferro. Instituto de Biología Universidad Nacional Autónoma de México, México, D. F. 2014. Consultado el 5 de diciembre de 2015 en: www.ibiologia.unam. $\mathrm{mx} /$.../Phengodidae

[10]. Buck J.B. The anatomy and physiology of the light organ in "Fireflies". Annals of the New York Academy of Sciences, 49 (3), 379-485, 1948. En: Davson H. A Textbook of General Physiology. Little, Brown and Company, Boston, 1964. Capítulo XXV, Bioluminiscence. $1086-1087$.

[11]. Timmins G. S., Roob F. J., Wilmot C. M., Jackson S. K., Swartz H. M. Firefly flashing is controlled by gating oxigen to light emitting cells. Journal of Experimental Biology, 204, 2795-2801, 2001.

[12]. Grenfield M. D. Missing link in firefly bioluminiscence revealed: NO regulation of photocite respiration. BioEssays 23 (11), 992995, 2001.

[13]. Seliger H.H., McElroy W.D. Spectral emission and quantum yield of firefly luminescence. Archives of Biochemistry and Biophysics. 88, 136-141, 1960. En: Davson H. A Textbook of General Physiology. Little, Brown and Company, Boston, 1964. Capítulo XXV, Bioluminiscence. 1095 - 1103.

[14]. Taylor B. N., Thomson A. The international system of units (SI). National Institute of Standards and Technology, special publication 330, Gaithersburg, MD 20899. 2008. pp 22. Consultado el 4 de febrero de 2014 en: http:/www.physics.nist. gov/Pubs/SP330/sp330.pdf

[15]. Karsten K., Kleiber J. Tratado de física. Editorial Gustavo Gili, S. A. $10^{a}$ Edición. 1945. Capítulo 14, 321-322.

[16]. Real Academia Española, Asociación de Academias de la Lengua Española. Diccionario de la lengua española, 23. ${ }^{\mathrm{a}}$ ed., Edición del Tricentenario, [en línea]. Madrid: Espasa, 2014.

[17]. Coblentz W.W. A physical study of the firefly. Gibson Bros. Washington, D.C. 1912. En: Levy H. C. Greatest Bioluminiscence, University of Florida Book of Insect Records. Chapter 29. 1998.

[18]. Harvey E.N. Bioluminescence. Academic Press, New York, 1952. En: Levy H. C. Greatest Bioluminiscence University of Florida Book of Insect Records. Chapter 29. 1998.

[19]. Seliger H.H., Buck J.B., Fastie W.G., McElroy W.D. The spectral distribution of firefly light. The Journal of General Physiology, 48 (1), 95-104, 1964

[20]. Frick-Ruppert J.E., Rosen J.J. Morphology and behavior of Phausis Reticulata (Blue ghost firefly). Journal of The North Carolina Academy of Science, 124, 139-147, 2008.

[21]. Lloyd J.E. Agressive mimicry in Photuris: firefly femmes fatales. Science, 149 (3684), 653-654, 1965.

[22]. Lloyd J. E. Firefly Semiosystematics and predation: A History. The Florida Entomologist, 73 (1), 51-66, 1990

[23]. Segelken R. Lured and liquidated, gullible male fireflies supply "Femmes fatales" with lifesaving chemical. Cornell Chronicle, Cornell University, Sept. 1, 1997. Consultado el 9 de diciembre de 2015 en: http://www.news.cornell.edu/stories/1997/09/ cornell-biologists-report-mimicry-and-murder-night.

[24]. Evans A. V. National Wildlife Federation field guide to insects and spiders and related species of North America. Sterling Publishing
Company. 2007. Consultado el 4 de febrero de 2014 en: http:/ www.nwf.org/Wildlife library/Invertebrayes/Firefly.aspx.

[25]. Stous H. A review of predation in Photuris, and its effects on the evolution of flash signaling in other. New World fireflies, Consultado el 6 de Julio de 2010 en: http:/www.colostate.edu/ Depts/Entomology/Courses/en507/papers_1997/stous.html.

[26]. Branham M. A., Greenfield M. D. Flashing males win mate success. Nature, 381 (6585), 745-746. 1996.

[27]. Strogatz S. Sync: the emerging science of spontaneous order. Capítulo I, Fireflies and the inevitability of sync. Hyperion Special Markets, 1-14. 2003

[28]. Carr K.F. A guide to integrating sphere radiometry and photometry, Labsphere inc. 231 Shaker Street, North Shutton, NH. USA. Parágrafo 4.1, 7.

[29]. Integrating Spheres. Sphere consideration, Spectral Products, 111 Higland Drive, Putnam, CT. 06260, USA. Consultado el 5 de febrero de 2014 en: http:/www.spectralproducts.com/spheres

[30]. Ryer A. Light measurement handbook. International Light, Inc. 1997. Capítulo 12, 58.

[31]. Rodríguez F. Diseño, construcción y puesta a punto de un radiómetro de esfera integradora $(400-700 \mathrm{~nm})$ Dpto. de Ingeniería de Circuitos y Sistemas E. U. de Ingeniería Técnica de Telecomunicación. Universidad Politécnica de Madrid. 2004. Consultado el 5 de febrero de 2014 en: http:/digital csic.es/ bitstream10261/11414/Proyecto\%20esfera.pdf

[32]. Panasonic selection guide, round type $3.0 \mathrm{~mm}$ visible light emitting diode, Panasonic Electronic Components. Optoelectronic devices. Consultado el 6 de febrero de 2014 en: http://www. bitsavers.org/components/panasonic/1990_Panasonic_ Optoelectronic_Devices.pdf

[33]. Hamamatsu Products. Si. Photdiode S1337 - 1010BR. Consultado el 6 de febrero de 2014 en: htpp:/hamatsu.com/us/en/ product/category/3100/4001/41003/S1337-1010BR/index.htlm

[34]. Texas datasheet. Low-noise JET-Input general purpose operational amplifier TL071. Consultado el 6 de febrero de 2014 en: http:/ti.com/product/t1071

[35]. National Research Council of the National Academies. Guide for the care and use of laboratory animals. The National Academies Press, Washington, D. C. 2001. Consultado el 21 de diciembre de 2015 en: https://grants.nih.gov/grants/olaw/Guide-for-the-Careand-use-of-laboratory-animals.pdf

[36]. Cardozo C.A., Mrad A. Aspectos técnicos y éticos en el cuidado y uso de modelos animales en Colombia. Consultado el 21 de diciembre de 2015 en: https://umshare.miami.edu/web/wda/ ethics/PABI.../Module14487.pdf

[37]. Cazamariposas Británica. Entomopraxis, Material Entomológico, pp. 29. Consultado el 6 de febrero de 2014 en: http:/entomopraxis. com/catalogo2012.pdf

[38]. Lloyd J. E. Flashes of Photuris fireflies: their value and use in recognized species. The Florida Entomologist, 52 (1), 29-35, 1969.

[39]. Saikia J. Changmai R. Baruah G. D. Bioluminiscence of fireflies and evaluation of firefly pulses in light of oscilatory chemical reactions. Indian Journal of Pure \& Applied Physics, 39, 825-828, 2001.

[40]. Lloyd J.E., Ballantine L.A. Taxonomy and behavior of Photuris trivittata sp. n. (Coleoptera: Lampyridae: Photurinae); redescription of Apisoma trilineata (Say) comb. n. (Coleoptera\ Lampyridae: LampyrinaelCratomorphini). The Florida Enthomologist, 86 (4), 464-473, 2003. 